Chapter 1

\title{
Electrospun Nanofiber Based Solid Phase Extraction
}

\author{
Samuel Chigome and Nelson Torto \\ Additional information is available at the end of the chapter \\ http://dx.doi.org/10.5772/57100
}

\section{Introduction}

The objectives of a sample preparation technique in Analytical Chemistry are twofold; transformation of a sample into a form that is suitable for instrumental analysis and to ensure that the sample is at the detection level for the instrument of choice. Solid phase extraction (SPE) is a popular sample preparation technique for liquid samples with subsequent chromatographic analysis [1]. SPE is employed with the aim of either reducing interferences or achieving analyte preconcentration in order to enhance instrumental detection. Although SPE can be described as a physical extraction process involving a liquid phase and a solid phase (that can be packed or free flowing sorbent), the increased use of packed sorbent formats seems to have led to a bias towards packed sorbent SPE devices [2]. One of the first applications of packed sorbent SPE was reported in 1951, when Braus and co-workers packed 1.2-1.5 kg of granular activated carbon into an iron cylinder for the isolation of organic compounds [3]. Since then, there has been significant progress in SPE technology, as evidenced by reports on new formats and sorbents covering a wide range of morphologies and chemistries [1].

SPE research and developments have progressed with a focus on SPE device fabrication or SPE method development. In principle, the heart of the SPE technique is the sorbent material as it has a direct influence on the selectivity, sorptive capacity and the format or the configuration of the resultant SPE device (s).

To predict and optimize extraction, it is important to be aware of the nature of the sorbent used with respect to physicochemical characteristics. The most important physicochemical characteristics for optimal extraction are porosity, specific surface area and surface chemistry.

Given that there will always be a need for new sorbent materials, it is imperative to focus research efforts on versatile sorbent fabrication techniques that could address current and anticipated challenges. Electrospinning is seen as having great potential as a sorbent fabrication technique, given its versatility [4]. 
The objective of the chapter is to equip the readers with sufficient knowledge that would enable them to fabricate and employ electrospun nanofibers as sorbents not only for SPE but for a wide range of applications. A discussion of the fundamental principles of SPE, the challenges associated with further research efforts and the unlimited potential that lies in electrospinning to address SPE will be presented. The use of electrospun nanofibers for SPE is an area that is still at its infancy (Scifinder scholar search of the words "Electrospinning" and"Solid Phase Extraction" showed 34 hits which consisted of 30 papers, 2 review articles, 2 patents and 0 books/book chapters) thus the chapter will be presented mostly in a postulative manner on the basis of the experiments conducted in the authors' research lab as well as literature reports.

\section{Fundamental principles of sorbent extraction}

Sorption can be defined as a process by which a substance (sorbate) is sorbed (adsorbed or absorbed) on or into another substance (sorbent) [5]. In the sample preparation context, the term sorbent refers to the solid extracting phase, including solid-supported liquid phases upon which an analyte is retained. Schwarzenbach and co-workers [6] made a distinction between absorption meaning into a three dimensional matrix, and adsorption as meaning onto two dimensional surface. Figure.1. shows a schematic representation of analyte adsorption (analyte accumulation onto the sorbent surface) and absorption (analyte accumulation into the bulk of the sorbent) type extraction mechanisms.
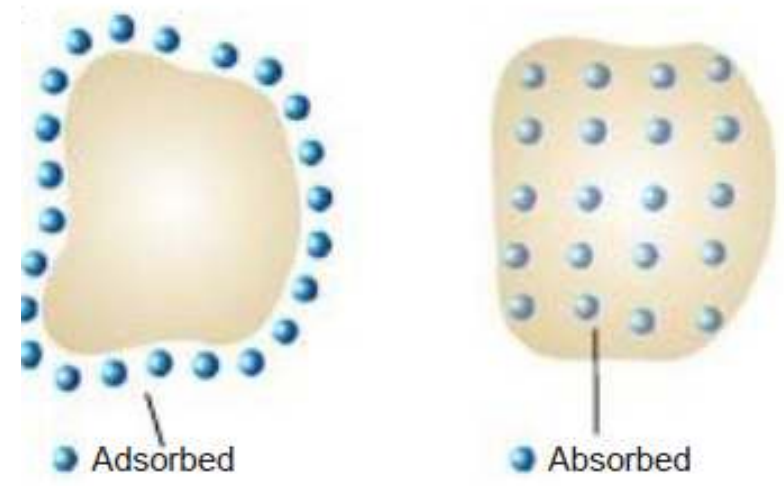

Figure 1. Schematic representation of (a) absorptive and (b) adsorptive extraction processes [7].

Although sorbent based extraction techniques could be classified on the basis of either adsorption or absorption, the two processes are not completely separable as they occur concurrently. In principle, what differs is the extent of contribution of the predominant extraction mechanism as that is solely dependent on the nature of the extraction phase. Consequently, it may be difficult to distinguish between the two processes experimentally [8]. 
thus the general term sorption is often used to refer to a combination of these processes. Despite the uncertainty of the extent of contribution of adsorption or absorption mechanisms in extraction, fundamentally, all sorbent based extraction techniques are guided by the thermodynamic partition or distribution coefficient $K$, which is usually expressed as the ratio of analyte concentration in the sorbent phase, $C_{\text {sorbent }}$ to that in the sample phase, $C_{\text {sample }}$;

$$
K=\frac{C_{\text {sorbent }}}{C_{\text {sample }}}
$$

For adsorption, surface structure (porosity and surface area governing the available sites for analyte retention) is the more important sorbent characteristic compared to chemical composition. While the chemical composition (governing the diffusion coefficient of the analyte into the sorbent) of the liquid phase is the more important for absorption.

Sorption from the sample phase is essentially a dynamic process in a heterogeneous system in which transport of the analytes between the sorbent and the sample phase is achieved. The process proceeds by a decrease in free energy until it reaches the minimum value (that is equilibrium). The mechanism of analyte adsorption or absorption is governed by the characteristics of interactions between the analyte and active sites of the sorbent. Therefore, sorbent selection is based on the binding mechanisms between the sorbent and analyte of interest. Table.1. shows different interaction mechanisms with their corresponding energies.

\begin{tabular}{ll}
\hline Interaction mechanism & Energy $(\mathbf{k J} / \mathbf{m o l})$ \\
\hline Van der Waals & $1-5$ \\
\hline Dipole-induced dipole & $2-7$ \\
\hline Dipole-dipole & $5-10$ \\
\hline Hydrogen bonding & $5-10$ \\
\hline lonic & $50-200$ \\
\hline Covalent & $100-1000$ \\
\hline
\end{tabular}

Table 1. Energies of interaction mechanisms [9].

The process of analyte sorption can be assumed to consist of multiple steps. Any of the steps may become rate limiting in controlling sorption of an analyte. The analyte may interact with a sorbent in at least four ways:

1. Through absorption, the analyte may interact with the sorbent by penetrating its three dimensional structure. Three dimensional penetration into the sorbent is a particularly dominating process for solid supported liquid phases. In the absorption process, analytes do not compete for sites; therefore, absorbents can have a high capacity for the analyte.

2. The analyte may interact two dimensionally with the sorbent surface through adsorption due to intermolecular forces [10]. Surface interactions may result in displacement of water 
or other solvent molecules by the analyte. In the adsorption process, analytes may compete for sites; therefore, adsorbents have limited capacity. Three steps occur during the adsorption process on porous sorbents; (a) film diffusion (when the analyte passes through a surface film to the solid phase surface), (b) pore diffusion (when the analyte passes through the pores of the solid phase), and (c) adsorptive reaction (when the analyte binds, associates or interacts with the sorbent surface) [11].

3. If the analyte is ionisable in aqueous solution, there may be an electrostatic attraction between the analyte and the charged sites on the sorbent surface. Sorbents specifically designed to exploit these types of ionic interactions are referred to as ion-exchange (either anion or cation exchange).

4. It is possible that the analyte and the sorbent may be chemically reactive toward each other such that the analyte becomes covalently bonded to the sorbent. This type of sorption is generally detrimental to analytical recovery and may lead to slow or reduced recovery $[6,11]$.

For porous sorbents, most of the surface area is inside the nanopores of the sorbent (see Fig. 2.). Nanopores of the sorbent are classified into three as; micropores (diameters smaller than 2 $\mathrm{nm}$ ), mesopores (2 to $50 \mathrm{~nm}$ ), and macropores (greater than $50 \mathrm{~nm}$ ) [12]. Most of the surface area is derived from the small diameter micropores and the medium diameter mesopores. Porous sorbents vary in pore size, shape, tortuosity and are characterized by properties such as particle diameter, pore diameter, pore volume, specific surface area and particle distribution.

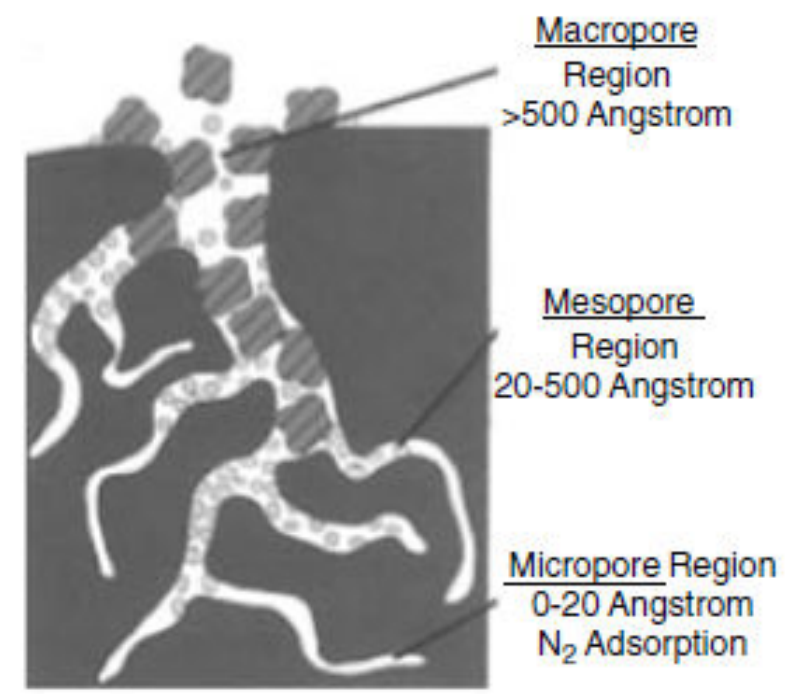

Figure 2. Schematic representation of porous regions of a sorbent [13]. 


\subsection{Solid phase extraction process}

Classically, batch mode liquid solid extractions were used in which the liquid sample was placed in contact with the bulk free flowing solid extracting phase. Equilibrium between the two phases was allowed to occur, followed by physical separation (decanting or filtering). Advancements of liquid solid extractions could be said to have taken two approaches; the first being solid phase microextraction (SPME) which consists of a two-step process in which the sorbent or solid supported sorbent is allowed to reach equilibrium before analyte desorption [14-17]. Figure.3. shows the most widely used version of SPME which is based on an organic polymer coated fused silica fiber operated in a syringe format. The syringe is designed to move the fiber in and out of the needle, which allows exposure of the fiber during extraction and desorption [18].

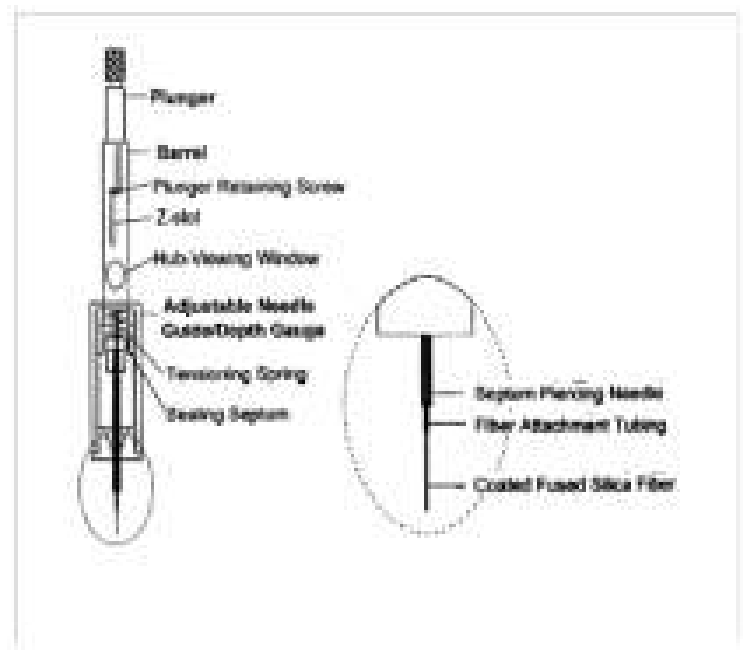

Figure 3. Schematic representation of the components of a commercially available SPME device [18].

While packed sorbent format SPE generally involves four steps;

Conditioning: functions to activate or "wet" the sorbent to prepare for its interaction with the analyte. This is especially necessary for hydrophobic sorbents that would not be activated by an aqueous sample. If the sorbent is not adequately conditioned, poor analyte retention may be achieved. If $\mathrm{pH}$ is critical for retention, then the conditioning solvent has to be matched to that of the sample to prepare for maximum analyte retention during the loading step.

Loading: when the liquid sample is added to the sorbent, sufficient residence time should be allowed for maximum analyte-sorbent interaction. This is especially critical when employing ion exchange to provide for adequate residence time of the sample solution in the sorbent since the analyte has to achieve an appropriate orientation for electrostatic retention with the sorbent functional groups. 
Washing: this serves to remove interferences retained on the sorbent leaving behind the analytes of interest. In some cases, it could be used to wash-off the analyte of interest while retaining the interferences. In mechanisms employing ion exchange, the $\mathrm{pH}$ of the wash solvent should be sufficient enough to disrupt the charged sites of interferences but not affect the analyte.

Elution: the elution solvent should be strong enough to disrupt all analyte-sorbent interactions in order to obtain the highest recoveries. However, there is a limit to the strength as harsh solvents would not only desorb analytes from the sorbent, but also strip strongly retained interferences.

Figure.4. shows schematic representations of the four approaches typically taken in practical SPE applications. Figure.4. (a) shows a three step SPE process in which; (1) the sorbent is conditioned after which the sample is loaded and finally (2) the analyte is eluted with the interferences being retained (clean up) or the analyte is simply eluted into a smaller sample volume (preconcentration).

Figure.4. (b) shows a four step SPE process in which; (1) the sorbent is conditioned after which the sample is loaded, (2) the interferences eluted and finally (3) the analyte is eluted (clean up) or the analyte is simply eluted into a smaller sample volume (preconcentration).

Figure.4. (c) shows a five step SPE process in which; (1) the sorbent is conditioned after which the sample is loaded, (2) the interferences eluted, (3) a fraction of analyte is selectively eluted and finally (4) the second fraction of analytes is eluted.
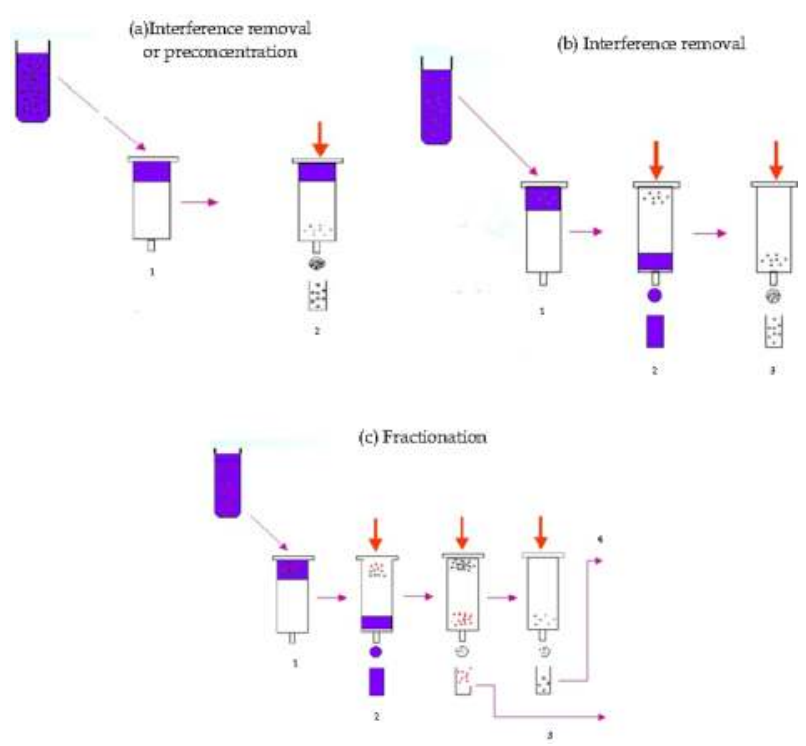

Figure 4. Schematic representation of practical SPE operations 


\section{Nanofiber based sorbents}

Sorbent selection for SPE is normally guided by the ability of a material to retain analytes selectively as well as facilitate rapid and complete elution. The sorption process must be reversible $[19,20]$. In addition to reversible sorption, SPE sorbents should possess a large specific surface area as well as exhibit stability in the sample matrix and elution solvents. Lastly, a sorbent material with a good surface contact with the sample solution would be most preferred.

Poole and co-workers [21] categorized SPE sorbents into three as; (i) general purpose, (ii) class specific and (iii) analyte specific. The most common retention mechanisms in SPE are based on van der Waals forces, $\pi-\pi$ interactions, hydrogen bonding, dipole-dipole interactions and ion exchange interactions. As a result, sorbents can be classified on the basis of retention mechanisms as; (i) reversed phase (ii) normal phase and (iii) ion exchange. From the materials perspective, sorbents are classified into three as; (i) carbon based (ii) inorganic based and (iii) polymer based.

The SPE sorbent fabrication technique of choice could be viewed as one that produces a material that exhibits chemical and morphological properties that can be easily modified. Similarly, an optimal SPE sorbent material combines the following benefits: (i) small diameter, (ii) large specific surface area, (iii) simplified fabrication/synthesis, (iv) ability to be modified in order to incorporate all sorbent chemistries/functionalities, (v) ability to be modified in order to incorporate all sorbent morphologies (vi) a material that can be packed in the lower (less than 10) $\mathrm{mg}$ range without presenting a backpressure limitation or low analyte recoveries.

The advent of nanotechnology has been a major leap forward in the research area of sorbent based sample preparation techniques as it opened up possibilities for a new class of materials that could be used in SPE applications. The main benefit of nanostructured materials is their large specific surface area that facilitates the miniaturization of SPE allowing for the use of a reduced sorbent bed mass that achieves high extraction efficiency.

Although nanoparticles offer improved performance as sorbent material [22], they inherently exhibit some limitations. One of the main challenges is associated with their handling in packed SPE formats.

Given the fact that nanoparticles have shown excellent properties as sorbent material, it would seem prudent to focus developmental efforts on nanostructured material with the aim to address the challenges of nanoparticles. This brings about a need for an alternative fabrication approach that carries with it the benefits of nanoparticles at the same time addressing some (if not all) of their limitations. From our research group's perspective, the use of electrospun nanofibers as sorbent material is seen as a possible way of carrying along the benefits of nanoparticles, whilst addressing their limitations and increasing the possibilities for modifying sorbent morphology and functionality. It is upon this background that the section has been compiled to present and discuss relevant examples that demonstrate the potential of electrospinning as an alternative fabrication technique. 


\subsection{Range of sorbent materials}

For it to be a good alternative, it should be able to fabricate a wide range of materials on the basis of type (inorganic, organic, inorganic/organic hybrid and biological), stability (mechanical and chemical) and morphology (pore structure and surface area).

\subsubsection{Carbon based}

Commercial polyacrylonitrile (PAN) based carbon fibers account for nearly $90 \%$ of the total carbon fiber output worldwide due to the high carbon yield and easy carbonization process. Therefore, PAN is mostly chosen as the precursor polymer for the preparation of electrospun carbon nanofibers (CNFs) [23].

Porous carbon nanofibers fabricated by thermal activation of electrospun PAN based CNFs have improved sorptive properties due to their large specific surface area. In a report by Oh and co-workers, a study was carried out to determine the toluene adsorption capacity for PANbased steam activated carbon nanofibers (ACNFs) [24]. The CNFs were activated between $800^{\circ} \mathrm{C}$ and $1000^{\circ} \mathrm{C}$ in order to evaluate the effect of activation temperature on fiber porosity. A relatively large adsorption capacity (65 g toluene/100 $\mathrm{g}$ ACNFs) was achieved by ACNFs (activated at $1000^{\circ} \mathrm{C}$ ) with a specific surface area of $1403 \mathrm{~m}^{2} \mathrm{~g}^{-1}$ as compared to (40 $\mathrm{g}$ toluene/100 $\mathrm{g}$ ACNFs) that was achieved for ACNFs (activated at $800^{\circ} \mathrm{C}$ ) with a lower specific surface area of $853 \mathrm{~m}^{2} / \mathrm{g}$. The results obtained in the study clearly demonstrated the great potential of steam activation as a post electrospinning modification approach for the fabrication of carbon nanofibers with a large sorptive capacity that could be used as sorbent material for SPE.

Shim and co-workers, compared the adsorption properties of electrospun steam activated carbon nanofibers and commercially available activated carbon fibers (CFs) [25]. Although there was a significant attenuation in the average fiber diameter from $20 \mu \mathrm{m}$ (CFs) to $250 \mathrm{~nm}$ (ACNFs), the change in specific surface area was not significant $\left(1015 \mathrm{~m}^{2} / \mathrm{g}\right.$ for CFs to $1193 \mathrm{~m}^{2} /$ $\mathrm{g}$ for ACNFs). Nevertheless, ACNFs exhibited a much larger adsorption capacity and faster adsorption/desorption kinetics due to their large number of shallow micropores and a more homogeneous surface, all these properties brought about by their nanoscale size. As demonstrated by the results obtained in the study, it was interesting to note that, besides the specific surface area, the pore structure and surface homogeneity also plays a significant role in improving sorptive capacity.

Bui and co-workers explored an alternative approach for fabricating porous carbon nanofibers in which PAN/pitch blends were electrospun with subsequent steam activation [26]. The specific surface area of the fabricated ACNFs increased from $723 \mathrm{~m}^{2} / \mathrm{g}$ (activation temperature $700^{\circ} \mathrm{C}$ ) to $1877 \mathrm{~m}^{2} / \mathrm{g}$ (activation temperature $900^{\circ} \mathrm{C}$ ). This was attributed to an increase in the mesopore fraction as the micropore fraction decreased. Given the fact that a specific surface area of $1877 \mathrm{~m}^{2} / \mathrm{g}$ is among the highest ever reported for nanostructured materials, it is expected that carbon nanofibers fabricated in the study would exhibit an excellent sorptive capacity. 


\subsubsection{Silica based}

Over the years, silica microparticles have been used widely as a sorbent for solid phase extraction. Recently, the focus has shifted to the use of silica nanoparticles due to their large specific surface area and intrinsic surface reactivity [27]. However, the use of silica nanoparticles for packed sorbent SPE still remains a challenge chiefly because of high back pressure, which explains why to date the smallest microparticle that has been used for packed sorbent $\mathrm{SPE}$ has a diameter of $8 \mu \mathrm{m}$ [28]. Due to the fact that the simplest electrospinning set-up allows the collection of nanofibers in the form of a porous nonwoven mesh, it follows that porous electrospun nanofiber based SPE sorbent formats could be easily fabricated. Therefore, it is anticipated that electrospun silica nanofibers would be used for packed sorbent SPE, thus overcoming the high back pressure limitation associated with silica nanoparticles.

The fabrication of inorganic nanofibers typically involves the electrospinning of a polymer/sol composite and subsequent calcination of the electrospun fibers. In 2002, Shao and co-workers were the first to report the fabrication of silica nanofibers [29]. The experimental approach involved first, the preparation of a silica sol from tetraethyl orthosilicate (TEOS), $\mathrm{H}_{3} \mathrm{PO}_{4}, \mathrm{H}_{2} \mathrm{O}$ followed by electrospinning of a PVA/silica sol. The PVA/ silica fibers were calcined to remove PVA resulting in amorphous silica fibers.

In 2003, Choi and co-workers reported a simplified approach in which silica nanofibers were fabricated directly from a silica sol [30]. Their fabrication method involved the preparation of a silica sol from TEOS, distilled water, ethanol and HCL with subsequent electrospinning. An interesting aspect of the fabrication approach was the fact that unlike in the first report by Shao and co-workers [29], TEOS did not contain a polymer to help spinnability, thus there was no need for the calcination step. Spectroscopic characterizationof the silica nanofibers confirmed the extensive hydrolysis of the TEOS suggesting the availability of a substantial amount of silanol groups for silylation [30].

A way of improving the adsorption capacity of silica nanofibers is to increase the specific surface area as a function of the pore volume. Wei and co-workers fabricated porous silica nanofibers containing catalytic silver nanoparticles [31]. TEOS, poly [3- (trimethoxysily) propylmethacrylate] (PMCM) and $\mathrm{AgNO}_{3}$ were used as precursors for the production of silica/ polymer hybrid nanofibers. On heat treatment of the electrospun fibers, degradation of the PMCM polymer resulted in pores that led to the increase in specific surface area from $11 \mathrm{~m}^{2} / \mathrm{g}$ to $600 \mathrm{~m}^{2} / \mathrm{g}$. The porous fibers exhibited an improved catalytic activity due to the increased surface area. Therefore it is expected that a similar approach could be used to improve the adsorptive capacity of silica fibers in SPE applications.

\subsubsection{Polymer based}

Synthetic polymers are the most popular class of materials that have been electrospun for SPE applications. Some of these polymers include polystyrene or polystyrene copolymers,[32-41] Nylon 6,[42-44] and poly (ethylene terepthalate) [45]. Other reports have appeared in the Chinese database, unfortunately the polymeric material was not described in English [46-48]. It is expected that synthetic polymers will continue to be the most popular class of electrospun 
materials for use as SPE sorbents. This is due to the fact that, of all the materials that have been electrospun, polymers show the greatest potential for tuning of the sorptive chemistries.

Sorptive capacity of polymer nanofibers could be improved by increasing the pore volume. There are several methods that have been reported for introducing porous structures in nanofibers which include electrospinning of polymer blends, [49] controlled humidity, [50] the salt induced process [51] and silica nanotemplating [52]. Silica nanotemplating is a relatively simple process compared to the other methods as they require handling of complicated interactions between the polymer matrix and pore generator. In addition, porosity and pore size of the resultant porous nanofibers may be controlled easily by adjusting the content and size of silica nanoparticles.

Shi and co-workers fabricated porous nylon 6 nanofibers using silica nanoparticles as the nanotemplate [52]. The experimental approach involved the fabrication of nylon 6/silica nanofiber composites, followed by removal of the silica nanoparticles through treatment with hydrofluoric acid. After the removal of the silica nanoparticles, the specific surface area and pore volume increased from $4.68 \mathrm{~m}^{2} / \mathrm{g}$ to $8.31 \mathrm{~m}^{2} / \mathrm{g}$ and $0.0133 \mathrm{~cm}^{3} / \mathrm{g}$ to $0.0250 \mathrm{~cm}^{3} / \mathrm{g}$ respectively. The increase in specific surface area demonstrated that the approach could be used to improve the sorptive capacity of nylon nanofibers for SPE applications.

\subsection{Control of selectivity}

Selectivity of sorbent material is a parameter of great concern in current SPE applications. Over the years, various sorbent modification strategies have been employed to impart selectivity based on basicity, acidity, polarity, size and more recently molecular recognition [53-55]. For it to be a good alternative, electrospinning should be able to allow the incorporation of an unlimited range of functionalities in order to target a broad spectrum of analytes.

\subsubsection{Polarity based}

Besides the conventional small functional groups like sulphonates and carboxylic acids, macromolecules can be incorporated into polymer nanofibers to improve their selectivity as SPE sorbents. Wan and co-workers reported the fabrication of porphyrinated nanofibers by copolymerization and electrospinning [56]. The fabrication approach involved solution copolymerization of acrylonitrile with vinyl porphyrins (see Scheme.1) and subsequent electrospinning of the resulting porphyrin copolymers.

\subsubsection{Ion exchange based}

At the beginning of 2008, Kang and co-workers reported a comparative study of the performances of poly (styrene-co-methacrylic), poly (styrene-co-p-sodium styrene sulphonate) and polystyrene nanofibers for the extraction of steroidal compounds [35]. Of the three kinds of nanofibers, those of poly (styrene-co- $p$-sodium styrene) exhibited the highest extraction efficiencies, while those of polystyrene were the least efficient. The trend was attributed to the fact that the polar model analytes favored the polar sorbent. With respect to applications, the study provided a platform for different chemistries that may introduce selectivity based on 


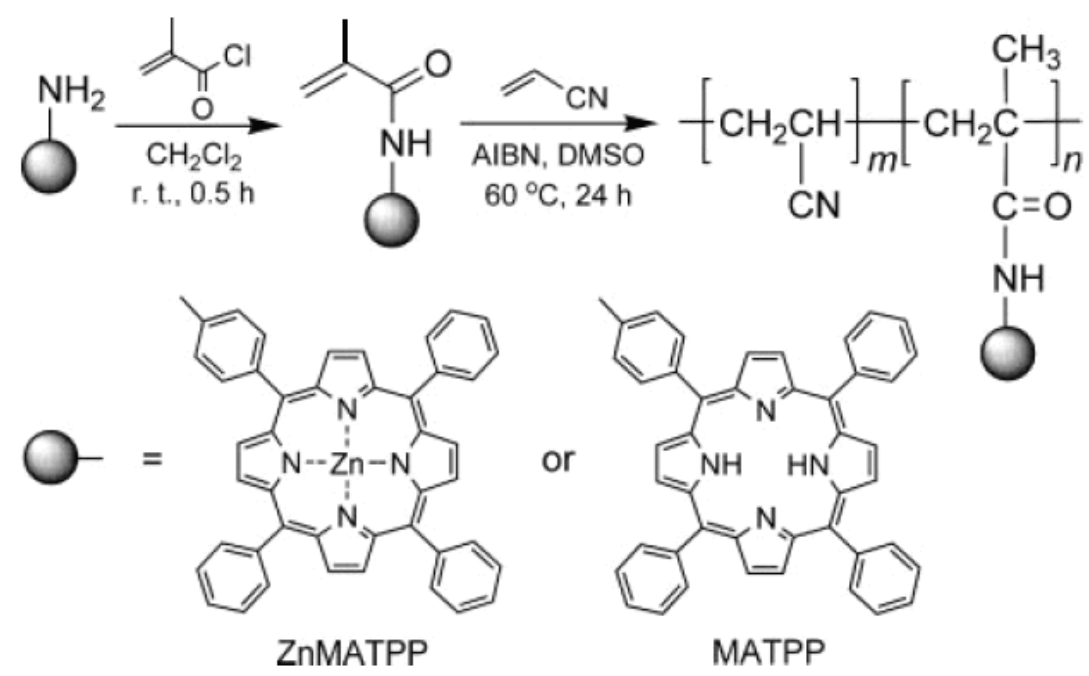

Scheme 1. Schematic representation of synthesis and molecular structure of the porphyrin copolymers [56].

hydrophobicity (polystyrene nanofibers) for non polar analytes, strong cation exchange properties (poly (styrene-co- $p$-sodium styrene sulphonate) nanofibers) for basic and neutral analytes and weak cation exchange properties (poly (styrene-co-methacrylic) nanofibers for strongly basic analytes. Due to the ease of spinnability of polystyrene copolymers and the demonstrated performance of the resultant fibers as SPE sorbents, a wide range of functionalities can be introduced on the polystyrene backbone by an experimental approach that involves copolymerization and electrospinning. It is expected that in the near future, SPE sorbents based on electrospun polystyrene copolymers will increase as there is a wide range of vinylic monomers that can be copolymerized with styrene.

\subsubsection{Molecular imprinting based}

Of all SPE sorbent materials that have been reported to date, those fabricated via the molecular imprinting technology have shown the best selectivity after immunosorbents [57].

Through electrospinning it is possible to incorporate the selectivity of MIPs either by encapsulating MIP nanoparticles into electrospun nanofibers or by imprinting the electrospun fibers.

Yoshimatsu and co-workers encapsulated molecularly imprinted nanoparticles into poly (ethylene terephthalate) (PET) nanofibers through electrospinning [45]. The composite nanofibers (Fig.5.) were used as a sorbent material for batch solid phase extraction of propanolol. As confirmed by radio ligand binding analysis, the specific binding sites in the composite nanofibers remained easily accessible and were chiral-selective. Furthermore, it was demonstrated that without the electrospun nanofiber based solid phase extraction step, the existence of propranolol residues in water could not be confirmed even with the sensitivity of HPLCMS/MS analysis [45]. 


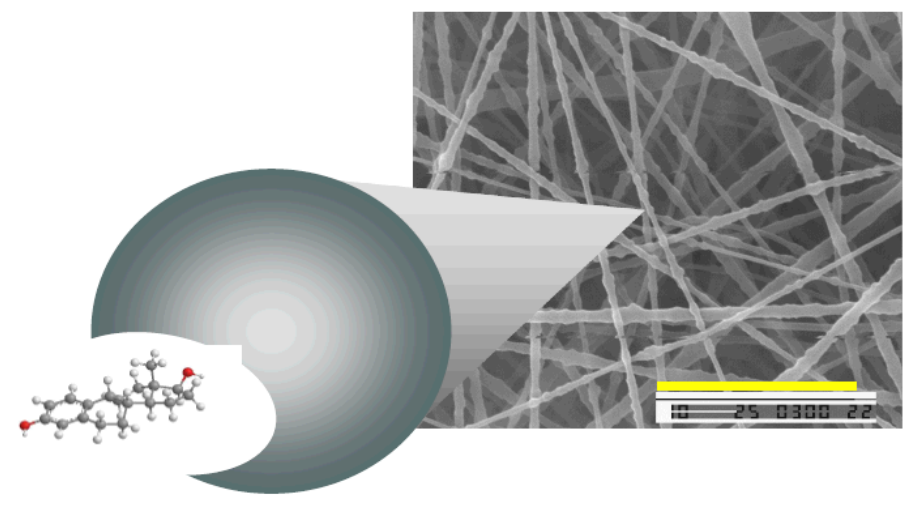

Figure 5. SEM image of electrospun nanofiber composite membrane containing molecular imprinted nanoparticles [45].
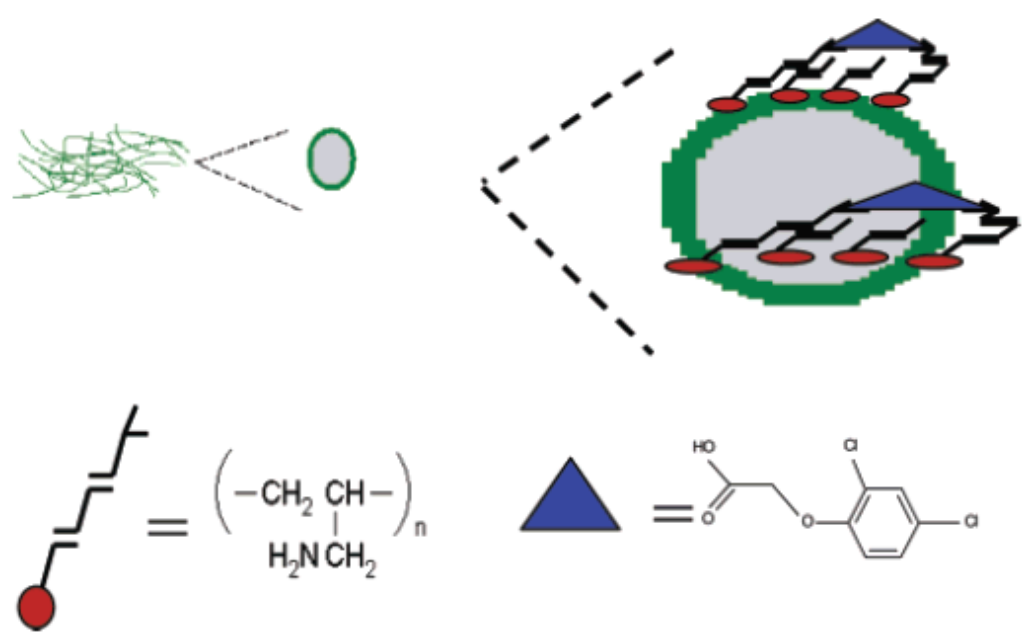

Figure 6. Schematic representation of molecularly imprinted nanofibers with binding sites specific for 2,4-D template molecules [58].

Chronakis and co-workers reported a simplified approach that allowed the generation of template defined sites directly during electrospinning [58]. The electrospun nanofibers were prepared from a solution mixture of PET and polyallylamine in the presence of a template molecule, 2,4-dichlorophenoxyacetic acid (2,4-D). Polyallylamine was used to provide functional groups that interacted with the template during the electrospinning process, and PET was used as the supporting matrix to ensure easy fiber formation and to minimize the conformational change of the polymers when the nanofibers were subjected to different 
solvent treatments. Figure.6. shows a schematic representation of a possible binding site model for the reported 2,4-D imprinted nanofibers.

\section{Nanofiber based SPE method development}

What is the starting point in electrospun nanofiber based SPE method development? The questions can be classified into four as;

i. Nature of the analyte: What are the functional groups on the analyte? What is the $\log P$ of the analyte? What is the pKa of the analyte? What is the solubility of the analyte?

ii. Nature of the electrospun fibers: How does one introduce a functionality of the target analyte onto the surface of electrospun fibers? Which polymer (s) or spinnable precursor (s) material should be employed? Which electrospinning protocol should be adopted (electrospinning conditions and electrospinning set-up)? Is there a need to modify the morphology of the fibers, if there is, how could it be done? What diameter of the fibers should be fabricated?

iii. Nature of the SPE device: What is the best way of handling the fibers (that is what sort of SPE device (s) should be fabricated)? What is the best way of packing the nanofibers (that is what shape or format)? How much sorbent should be packed?

iv. SPE operation: What sample volume, analyte concentration, volume of SPE solvents and at what flow rate?

Of all these, the most important aspect is to come up with a feasible electrospun nanofiber based SPE device as that serves as a platform for a optimal SPE method development.

To date, fabricated SPE devices that employ electrospun nanofibers as a sorbent bed have been based on polystyrene or nylon polymers. This has resulted in a classification of electrospun nanofiber based SPE devices into two as: polystyrene type (polymer fibers of a relatively low mechanical strength); and, nylon type (polymer fibers of a relatively high mechanical strength) [59].

\subsection{Polystyrene type SPE devices}

In 2007, Kang and co-workers were the first to report the use of electrospun polymer nanofibers for packed sorbent SPE [60]. They manually packed $1 \mathrm{mg}$ of polystyrene nanofibers into a 200 $\mu \mathrm{l}$ micro pipette tip to form a micro column as shown in Fig.7. (a) and (b) as published in their 2009 article. Solvents were pushed through the electrospun nanofiber based SPE device manually by the pressure of air forced by a gas tight plastic syringe $(2 \mathrm{~mL})$ (Fig.7 (c)). The device demonstrated a leap forward regarding the use electrospun nanofibers for miniaturized SPE devices. Although the packing process involved the use of simple homemade tools, it seems the packing operation is not that simple as reproducibility relies very much on operator experience. Nevertheless, the study created a platform for further research as it clearly demonstrated that nanofibers allowed the miniaturization of SPE devices without compro- 
mising the extraction efficiency. The packing procedure took advantage of the ease of rolling polystyrene nanofibers into nanofiber clews. Several reports followed where either polystyrene or polystyrene copolymer nanofibers were employed as the sorbent bed packed in micro column format [61-66].
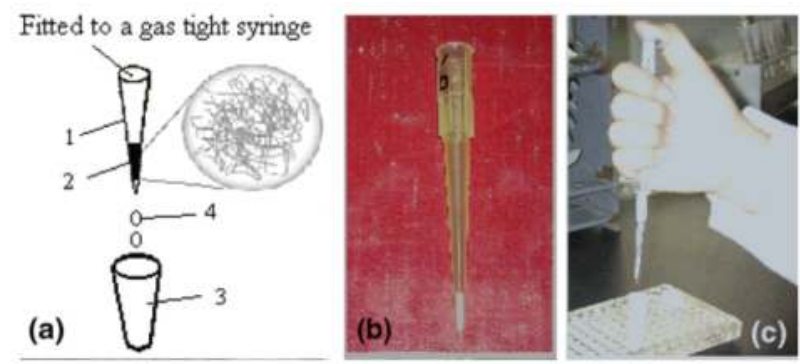

Figure 7. Micro column packed with polystyrene nanofibers and designed setup: (a) Schematic representation of packed fiber solid phase extraction device: (1) Pipette tip, (2) nanofibers packed, (3) eppendorf certrifuge tube, (4) desorption solution; and (b) Photograph of the micro-column; and (c) Photograph of a manual device [63].

All reports seem to have focused mainly on demonstrating the feasibility of using electrospun nanofibers as a SPE sorbent material since the experiments were biased towards obtaining maximum recovery.

For a better understanding of the use of electrospun nanofibers as a sorbent bed, it would be necessary to determine the mechanical strength, packing density and retention characteristics of the sorbent bed. In an effort to determine the mechanical strength of electrospun polystyrene fibers as a function of the effect of the force applied in the manual packing process, in our research group we viewed the top side of the micro column sorbent bed under scanning electron microscopy (SEM) (see Fig.8. (b-c)). The SEM morphology confirmed that the force applied did not induce any breakup or significant flattening of the electrospun polystyrene fibers.

One of the main drawbacks of the micro column packing procedure is consistency as it relies very much on the experience of the researcher. The main challenge is the rolling up of a uniform size of fiber clews. Without precise control of the size of fiber clews as well as the force applied, it would be difficult to maintain a uniform packing density and sorbent bed height, thus inconsistency of flow characteristics. Since electrospun nanofibers are applicable as a HPLC stationary phase, adopting this packing method for HPLC columns would be a challenge. This is in light of the fact that, without precise control of the packing procedure, band broadening could result due to multiple flow paths as a function of non uniform fiber orientation. Despite all these challenges, the development of the micro column packing procedure was invaluable to electrospun nanofiber based SPE sorbent research as it was used as a platform for evaluating the feasibility of using electrospun nanofibers as a sorbent bed.

Two important aspects in the development of SPE technology are simplicity of sorbent fabrication and miniaturization of devices. It is a fact that electrospun fibers could be packed 

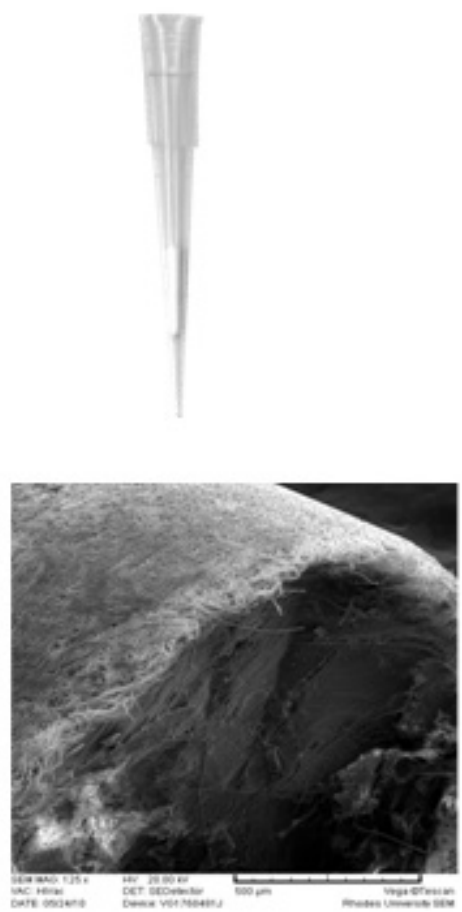
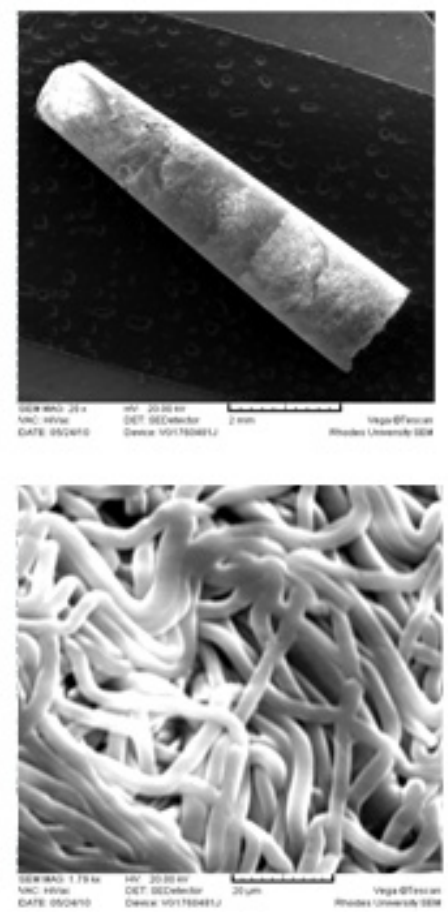

Figure 8. (a) Photograph of micro column SPE device, SEM images of (b) sorbent bed (c) magnification of top edge (d) zoomed in top surface.

in all existing formats and configurations for both SPE and SPME [4]. Furthermore, the use of electrospun fibers introduces an aspect of simplicity and miniaturization to the fabrication of SPE devices.

To date, methods of SPE disk fabrication involve a complicated multi step process in which microparticles are tightly held together within an inert fiber matrix, such as polytetrafluoroethylene [67]. This has limited the range of disk sorbent chemistries that are available. Two possible ways of simplifying the disk sorbent fabrication process would be; (i) to incorporate nanoparticles into nanofibers and pack the fibers in disk format or (ii) to fabricate nanofibers of the material with the chemistry of interest and pack them in disk format.

In 2010, our group reported an alternative polystyrene fiber based SPE device fabrication procedure [68]. The experimental approach consisted of copolymerization with subsequent electrospinning of the resultant polymer. $10 \mathrm{mg}$ of electrospun polystyrene fibers were packed in a disk format $(5 \mathrm{~mm} \times 1 \mathrm{~mm}$ ) (Fig.9.). The study clearly demonstrated how the use of electrospun fibers can simplify SPE disk fabrication as the sorbent material was packed using simple homemade tools. It is expected that in the near future, a wide range of chemistries will 
be introduced on disk SPE devices fabricated using a similar approach that will ultimately lead to a routine disk fabrication technology.

Although the procedure involved a manual compression stage, it was presumably simplified as it did not involve the rolling up of fiber clews. Unlike the micro column SPE device where some degree of flattening was observed, for this device, flattening was not observed as viewed under SEM (see Fig.9. b-c). This suggested that the force applied in the packing process was lower compared to that applied for the micro column SPE device. A possible explanation could be that the wire $(0.5 \mathrm{~mm}$ diameter) employed in the micro column SPE device fabrication procedure by virtue of having a smaller contact surface compared to the glass rod (5 $\mathrm{mm}$ diameter) employed in the disk SPE device fabrication procedure resulted in increased pressure.
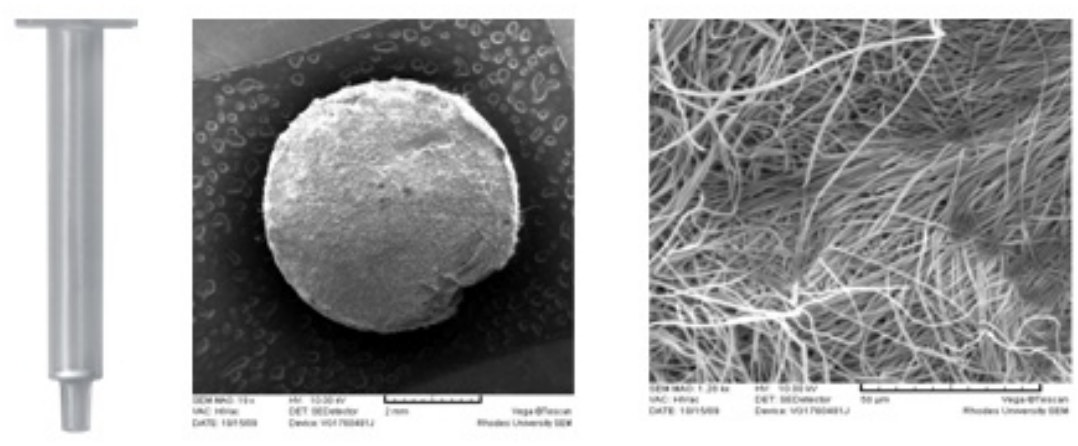

Figure 9. (a) Photograph of disk (I) SPE device, images of (b) sorbent bed (c) zoomed in top surface [68].

Despite the observed slight flattening of the electrospun polystyrene fibers in the micro column packing format, percolation of the solvents was not hindered which suggested that the packing procedure was adequate for fundamental experiments. To date, it seems, the only way of fabricating SPE devices that use electrospun nanofibers of a relatively low mechanical strength (as represented by polystyrene) would be via a mechanical compression stage. A possible way of improving on consistency could be to automate the mechanical compression stage.

\subsection{Nylon type SPE devices}

In 2009, Xu and co-workers were the first to report the fabrication of SPE devices that rely on relatively mechanically strong electrospun nanofibers as the sorbent bed [69]. Nylon 6 nanofiber sorbent beds were "packed" by cutting out circular portions $(1.5 \mathrm{~cm} \times 120-150 \mu \mathrm{m}$ $\times 1.5 \mathrm{mg}$ ) of the nanofiber sheet (see Fig.10.).

There have been several other reports on the use of nylon 6 nanofibers packed in the same format [70-74]. Similar to the first report by $\mathrm{Xu}$ and co-workers [69], all these reports seem to have focussed on demonstrating the feasibility of using electrospun nylon 6 nanofibers as a SPE sorbent material. 


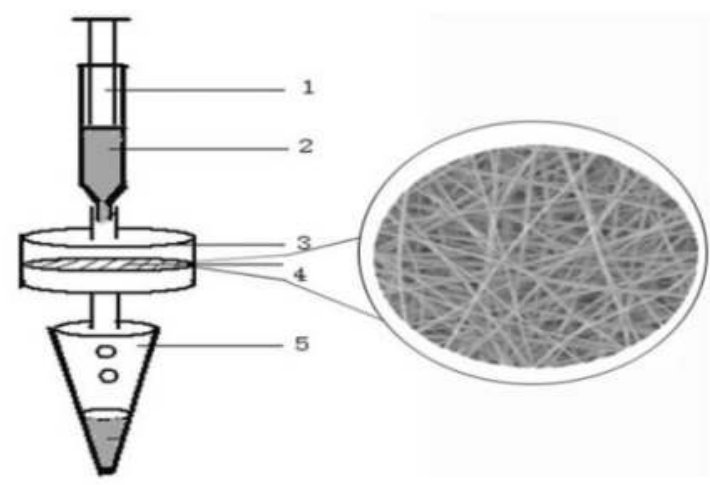

Figure 10. The fiber-filter solid phase extraction (SPE) device: gas tight syringe (1); sample solution (2); filter (3); electrospun mat using nylon 6 nanofibers (4); and, collecting tube (5) [70].

In 2011, Bagheri and co-workers reported an alternative fabrication procedure for a nylon type SPE device in which a polypyrrole-nylon 6 blend was employed as a sorbent bed [75]. Similar to the nylon 6 disk sorbent fabrication procedure, a nanofiber sheet $(1 \times 1 \mathrm{~cm})$ was cut out directly from the aluminium foil (see Fig.11.). However, the extraction procedure was not a flow through process as the sorbent bed was held by a wire and dipped into a sample solution. Although they referred to their device as micro SPE, it could be regarded as a SPME device as it was equilibrium based. Nevertheless, it is presented in this context as the fabrication procedure takes advantage of the mechanical strength of nylon nanofibers to illustrate the dependence of electrospun nanofiber evice fabrication on nanofiber mechanical stability.

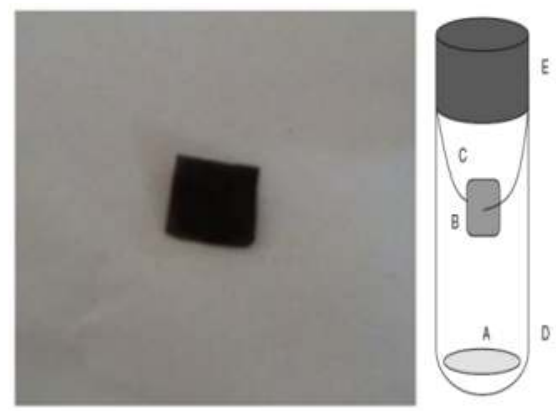

Figure 11. (a) Image of the polypyrrole-polyamide nanofiber sheet used for micro-solid phase extraction ( $\mu$-SPE). (b) Sample extraction: (A) magnetic bar; (B) nanofiber sheet; (C) thin holding wire; (D) vial; and, (E) vial cap [75].

The only report of electrospun nanofiber based micro extraction in a packed syringe (MEPS) was by Bagheri and co-workers [76]. Their packing procedure involved the manual compression of $8.1 \mathrm{mg}$ of polyrrole/nylon 6 nanofiber blend into a $1 \mathrm{~mL}$ insulin injection syringe. The electrospun nanofiber sorbent bed was laid flat at the bottom of the syringe barrel between 
two SPE frits. It is quite unfortunate that the authors did not publish the resultant SPE device otherwise it would have contributed towards a better understanding of the behaviour of the sorbent bed under compression. Similar to conventional MEPS [77] (see Fig.12.) procedure that proceeds via a draw eject cycle, a variable speed stirring motor was employed to drive solvents through the electrospun nanofiber MEPS device. Despite the fact that the authors did not publish their fabricated device, their contribution was invaluable as they were the first to demonstrate an electrospun nanofiber based MEPS device.

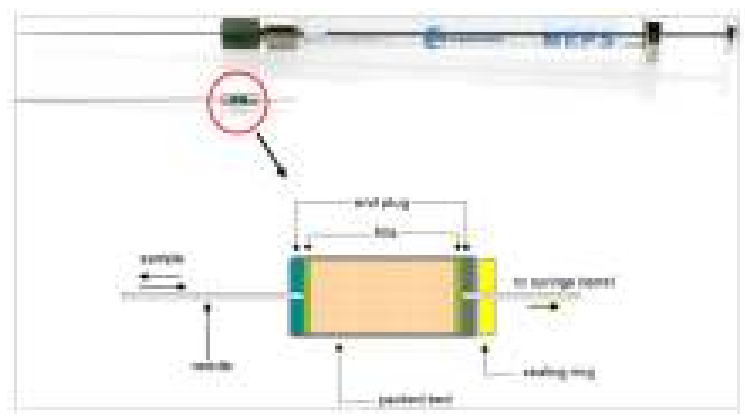

Figure 12. Representation of a MEPS syringe from SGE Analytical Science [77]

In our group, we fabricated a miniaturised version of an electrospun nylon 6 nanofiber based disk SPE device (Fig.13.a). The device was fabricated by cutting out circular portions of $5 \mathrm{~mm}$ diameter from the nanofiber sheet (Figure.13.b) and stacking them up to an optimal sorbent mass of $4.6 \mathrm{mg}(5 \times 350 \mu \mathrm{m}$ disks $)$.
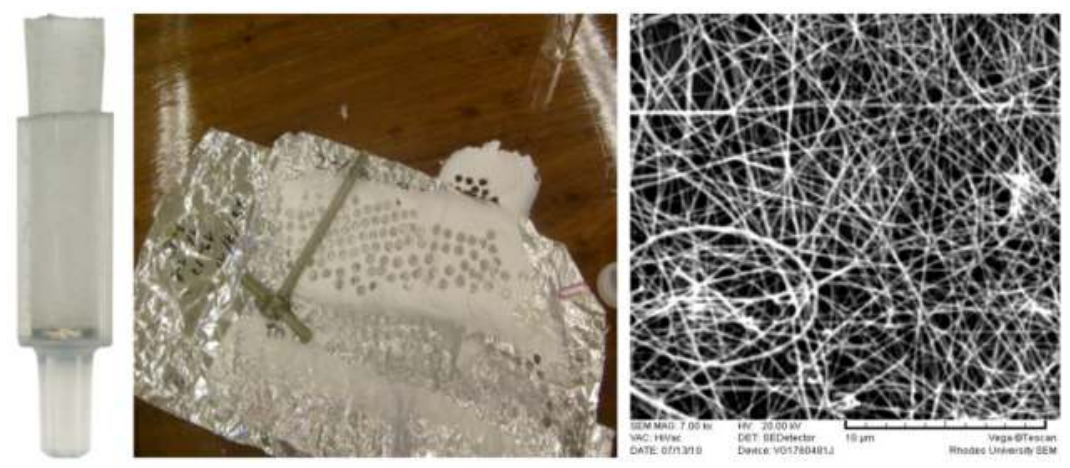

Figure 13. (a) Photograph of disk (II) SPE device, (b) photograph of an electrospun nylon 6 nanofiber mat showing regions from which disks were cut and (c) SEM image of sorbent bed zoomed in top surface [59].

Operationally, solvents have been driven through the SPE sorbent bed manually by a micropipette, syringe or by a vacuum manifold. All these approaches seem to be tedious particularly 
at the stage of determining the breakthrough volume. Simplification of the electrospun nanofiber based SPE process has been achieved in our group by employing a syringe pump for semi automation.

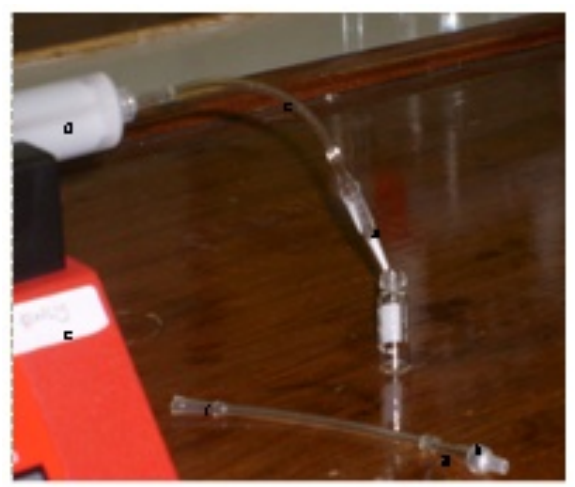

Figure 14. Syringe pump driven semi automated systems (a) micro column SPE device (b) modified disk (I) and disk (II) SPE device (s) (c) syringe pump (d) polypropylene syringe (e) PVC tubing (f) polypropylene adaptor (g) glass adaptor.

It is expected that in future, further simplification of electrospun nanofiber based SPE operation may be achieved employing robotic solid phase extraction systems. Despite the fact that robotic SPE systems like the Hamilton Micro Lab 2200 [20] and the Zymark Py Technology II ${ }^{\circledR}$ [21] achieved minimal success contrary to expectation, the knowledge gained could be useful as a step towards a new direction in automated electrospun nanofiber based SPE.
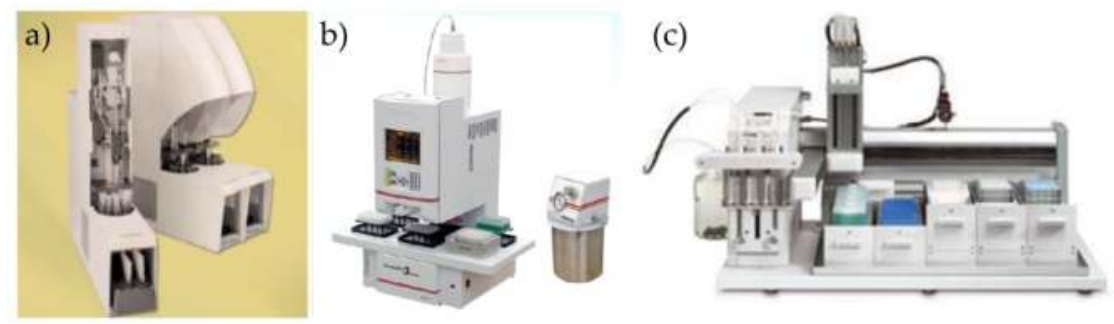

Figure 15. Most common robots for automated SPE (a)Zymark Rapid Trace System (b) Tomtec Quadra System (c) Gilson SPE 215 System.

\section{Theoretical aspects}

An optimal sorbent could provide a platform for fast analyte mass-transfer kinetics, which depends on the physicochemical properties of the sorbent (surface area, pore structure and 
surface chemistry). Although the physicochemical properties of the sorbent may be used to predict SPE performance, bias towards sorbent characterization in SPE-method development is more effective, as the contribution of physicochemical properties and flow rate of solution to analyze mass transfer kinetics is significant. Initial evaluation of new sorbent materials for SPE application can be achieved without complete characterization of the physicochemical properties of the sorbent. Progress could be made towards a better understanding of new sorbent materials by relying on the theoretical prediction of the physicochemical properties of the sorbent.

For sorbent screening, does one start off with batch experiments and then transfer to packed sorbent formats? Batch experiments are important as they give information about sorbent adsorption characteristics, in particular adsorption kinetics derived from the equilibrium isotherms (Freundlich and Langmuir) [78]. Although batch and flow through SPE rely on the distribution or partition coefficient, thus directly linked, a flow through based screening experimental approach that relies on breakthrough profiles could be employed.

Given that the major sorption parameters characterizing a sorbent are analyte recovery efficiency and breakthrough volume [21], the experimental design for fabrication and evaluation of SPE devices could proceed in two steps:

i. The first involves establishing an optimal sorbent mass, packing format and SPE method at which quantitative recoveries (preferably above $80 \%$ ) are achieved; and

ii. The second involves determining breakthrough curves from which sorbent retention characteristics are derived.

One of the most important characteristic parameters in establishing the suitability of a SPE sorbent bed for extracting target analytes is the breakthrough volume $\left(V_{B}\right)$ as it gives an indication of the sorbent's loading capacity. Assuming that there is a measurable analyte retention, the breakthrough curve forms a sigmoid shape (see Fig.17.) that gives an indication of the analyte mass transfer kinetics as a function of the sorbent retention characteristics [21]. In addition to the breakthrough volume, two important parameters that are obtained from the breakthrough curve are holdup volume $\left(V_{M}\right)$ and retention volume $\left(V_{R}\right)$.

These three parameters: $\left(V_{B}\right),\left(V_{R}\right),\left(V_{M}\right)$ can be defined as points corresponding (on the breakthrough curve) to $1 \%, 99 \%$ and $50 \%$ of the maximum concentration of analyte in the eluate. From these parameters, two important chromatographic characteristics of a sorbent bed; theoretical plates $(N)$ and retention factor $(k)$ are calculated.

The number of theoretical plates corresponds to the extraction efficiency and it depends on the physicochemical properties of the sorbent, particularly the available surface area for analyte retention. While the retention factor gives an indication of the quantity of sorbed analyte and it is directly related to the sorbent recovery efficiency.

Two methods have been employed for the calculation of theoretical plates for conventional SPE sorbents, the first was proposed by Werkhoheve-Goëwie and co-workers [79] Eq.2. 


$$
V_{B}=V_{R} *\left(\frac{\sqrt{N}-2}{\sqrt{N}}\right)
$$

While the second method was proposed by Lövkvist and Jönsson [80] who adopted a differential numerical solution to the breakthrough curve for analytes on a sorbent bed with a low number of theoretical plates Eq.3.

$$
V_{B}=V_{R}\left(a_{o}+\frac{a_{1}}{N}+\frac{a_{2}}{N^{2}}\right)^{\frac{-1}{2}}
$$

Where $a_{0}=(1-b)^{2}, a_{1}$ and $a_{2}$ are complex functions of $b$ evaluated from the tabular data [80] and $b$ is the breakthorugh level (the fraction of the total mass of analyte which has passed through the sorbent bed).

The retention factor $(k)$ is calculated from the fundamental equation of chromatography expressed as;

$$
V_{R}=V_{M}(1+k)
$$

Given that the equations for calculating $(N)$ and $(k)$ have been applied to microparticle based sorbents (commercially available sorbents), a question raised would be whether they would be suitable for nanofibrous sorbent bed material. Moreso, bearing in mind that the research work on electrospun nanofiber based SPE sorbents is a step towards the development of electrospun nanofiber based HPLC stationary phases, it is necessary to have a fundamental understanding of their kinetic and thermodynamic properties in relation to retention characteristics.

Theoretical and experimental methods have been proposed for determining breakthrough curves [81, 82]. Although experimental breakthrough curve determination by frontal chromatography (continuously added sample to sorbent bed see Fig.16.) is more tedious, it is more useful for SPE device fabrication. This is due to the fact that it serves as a guide for understanding the effect of sorbent packing format, packing density and sorbent morphology on the flow characteristics of the sample phase.

Under ideal conditions, the breakthrough curve forms a smooth sigmoid shape (see Fig.17.) with the specific shape depending on the sorbent retention characteristics

However, experimentally the breakthrough curve is plotted by using a line of best fit as the data points do not always follow a smooth pattern.

The correct determination of breakthrough parameters is subject to debate as several methods have been proposed $[83,84]$. Nevertheless, a mathematical modeling approach may be said to 

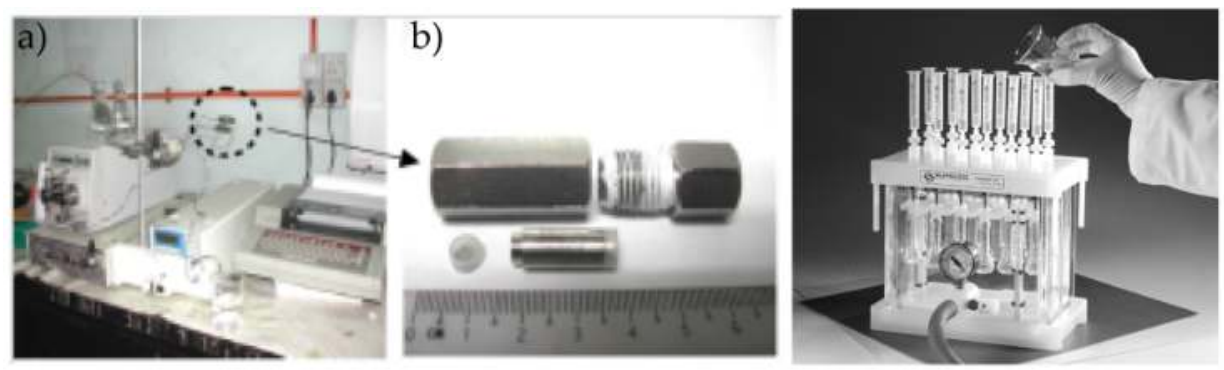

Figure 16. Representation of a (a) online (b) offline frontal chromatography set-up

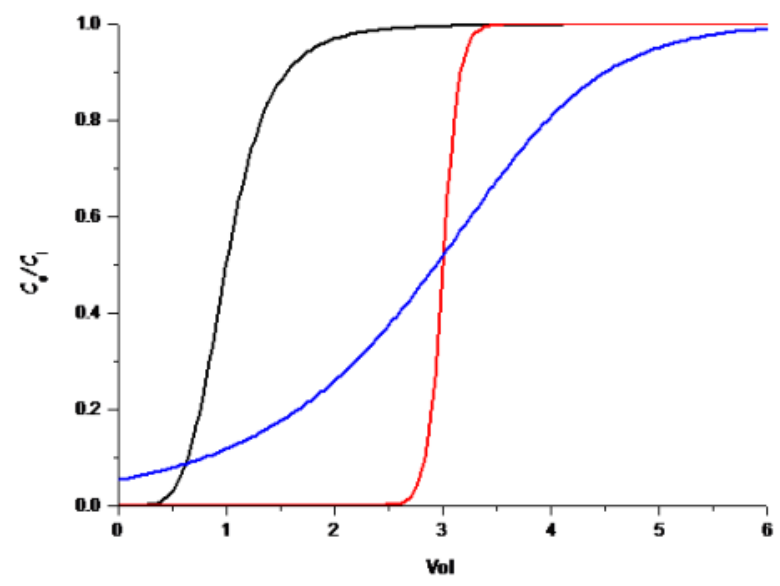

Figure 17. Mathematical simulations of breakthrough curves that can be obtained

be more preferable as it takes into consideration the actual shape of the breakthrough curve. In addition, interpreting observed experimental phenomena from the mathematical modeling perspective is invaluable as it provides a platform for linking up the various parameters influencing any system [85-89].

Our research group [68] and that of Susan Olesik were the first to report chromatographic parameters of electrospun nanofiber sorbent beds and chromatographic parameters of thin layer chromatography (TLC) plates [90], respectively. Therefore, an approach adopted in our group and results obtained will be discussed. Due to the importance of the accurate determination of breakthrough parameters, it was imperative for us to compare different mathematical models for each set of data points obtained in the experimental procedure.

A breakthrough curve typically follows a form of an "S" shape (Fig.18.). Therefore, it may be considered to be a form of a logistic function. A simple logistic function may be defined by the formula; 


$$
Y(x)=\frac{1}{1+e^{-x}}
$$

Where the variable $Y$ might be considered to denote analyte concentration, $e$ is Euler's number and the variable $x$ might be thought of as volume. For values of $x$ in a defined range of real numbers from $-\infty$ to $+\infty$, exponential growth which slows to linear growth and progresses to exponential decay is observed.

The logistic function is the solution of the simple first order non-linear differential equation

$$
\frac{d}{d x} Y(x)=Y(x)(1-Y(x))
$$

The qualitative behavior may be understood as follows; the derivative is 0 at $Y=0$ or 1 and the derivative is positive for $Y$ between 0 and 1 , and negative for $Y$ above 1 or less than 0 . Thus for any value of $Y$ greater than 0 and less than $1, Y$ grows to 1.

Alternatively, the logistic function may be expressed symbolically as;

$$
Y(x)=\frac{e^{x}}{e^{x}+e^{c}}=\frac{1}{1+e^{-x}}
$$

Where 1 is chosen as the constant of integration $\left(e^{c}=1\right)$

Besides the logistic function, sigmoid functions include the ordinary arctangent, the hyperbolic tangent and the error function. However, for this book contribution, the logistic function was employed as the fundamental equation upon which curve fitting models were developed. The curve fitting mathematical model depends on the "S" shape of the data points and the selection is guided by by the resulting regression constants.

The Boltzmann model has been used to fit experimental data for conventional SPE sorbents as it has generally been accepted as an accurate method [84]. Thus it was the first model that was explored (see equation 8).

$$
Y=A_{2}+\frac{A_{1}-A_{2}}{1+e^{\frac{x-x_{o}}{d x}}}
$$

Where $\mathrm{Y}$ represents the ratio of the eluted $\left(C_{e}\right)$ to the inlet $\left(C_{i}\right)$ analyte concentration $\left(\frac{C_{e}}{C_{i}}\right), x$ is the volume of sample flowing through the sorbent, $A_{1}$ and $A_{2}$ are two regression parameters. The maximum value of $\left(\frac{C_{e}}{C_{i}}\right)$ is $\left(A_{2}\right)$ and it is obtained when $x \rightarrow \infty$, while the minimum value 
of $\left(\frac{C_{e}}{C_{i}}\right)$ is approximately $A_{1}$, obtained for $x \rightarrow 0$. The retention volume which corresponds to the point of inflexion on the breakthrough curve was obtained from the model and it corresponds to the $\left(\frac{C_{e}}{C_{i}}\right)=\frac{A_{1}+A_{2}}{2}$

$$
V_{R}=x_{0}
$$

The hold-up volume $\left(V_{M}\right)$ and the breakthrough volume $\left(V_{B}\right)$ were calculated as the $x$ values obtained from equating the following;

$$
\begin{aligned}
& \left(\frac{99}{100}\right) * A_{2}=A_{2}+\frac{A_{1}-A_{2}}{1+e^{\frac{x-x_{0}}{d x}}} \\
& \left(\frac{1}{100}\right) * A_{2}=A_{2}+\frac{A_{1}-A_{2}}{1+e^{\frac{x-x_{0}}{d x}}}
\end{aligned}
$$

And by solving these equations, the formulae below were derived;

$$
\begin{gathered}
V_{B}=x_{0}+(d x)^{*} \ln \left(\frac{100}{99}\left(1-\frac{A_{1}}{A_{2}}\right)-1\right) \\
V_{M}=x_{0}+(d x)^{*} \ln \left(99-100^{*} \frac{A_{1}}{A_{2}}\right)
\end{gathered}
$$

The second mathematical model employed is the sigmoid three parameter model (see equation 14)

$$
Y=\frac{a}{1+e^{-\frac{\left(x-x_{0}\right)}{b}}}
$$

Similar to the Boltzmann model, $\mathrm{Y}$ represents the ratio of the eluted $\left(C_{e}\right)$ to the inlet $\left(C_{i}\right)$ analyte concentration $\left(\frac{C_{e}}{C_{i}}\right), x$ is the volume of sample flowing through the sorbent, $a$ and $b$ are two regression parameters. The maximum value of $\left(\frac{C_{e}}{C_{i}}\right)$ is $(a)$. Calculation of $V_{M}$ and $V_{B}$ was achieved by expressing $\left(\frac{C_{e}}{C_{i}}\right)$ as a fraction of $a$ as follows;

$$
\left(\frac{C_{e}}{C_{i}}\right)=F^{*} a
$$


Where $F=\frac{1}{1+e^{-\frac{\left(x-x_{0}\right)}{b}}}$ and solving for $x$ (corresponding to either $V_{M}$ or $V_{B}$ ) resulted in the expression below

$$
x=x_{0}+b \ln \frac{F}{1-F}
$$

$V_{M}$ and $V_{B}$ were obtained from equation 16 at $F$ values of 0.99 and 0.01 respectively.

The third model that was explored is the Weibull five parameter model (see equation 17)

$$
Y=Y_{0}+a\left[1-e^{-\left(\frac{x-x_{0}+b \ln 2^{\frac{1}{c}}}{b}\right)^{c}}\right]
$$

$Y_{o}$ and $a$ correspond to the maximum and minimum points on the breakthrough curve, $x_{o}$ corresponds to the point of inflexion while $b$ and $c$ are regression parameters.

As $x \rightarrow \infty, Y$ approaches the maximum value of $\left(\frac{C_{e}}{C_{i}}\right)=Y_{o}+a$ thus, $\left(\frac{C_{e}}{C_{i}}\right)$ at any point of the breakthrough curve can be expressed as a fraction $(F)$ of the maximum value of $\left(\frac{C_{e}}{C_{i}}\right)$.

If the maximum value of $\left(\frac{C_{e}}{C_{i}}\right)$ is expressed as

$$
F^{*}\left(Y_{0}+a\right)=Y_{0}+a\left[1-e^{\left.-\left(\frac{x-x_{0}+b \ln 2^{\frac{1}{c}}}{b}\right)^{c}\right]}\right.
$$

Solving for $x$ results in the expression below;

$$
x=x_{o}-b \ln 2^{\frac{1}{c}}+b\left[\ln \left[\left(\frac{Y_{o}}{a}+1\right)^{-1}(1-F)^{-1}\right]\right]^{\frac{1}{c}}
$$

Therefore, $V_{M}$ and $V_{B}$ can be calculated at $F$ values corresponding to $\frac{99}{100}$ and $\frac{1}{100}$ respectively.

An example in which we compared the Boltzmann model and the sigmoid Weibull five parameter model for the evaluation of a microcolumn SPE device. The experimental approach to determine the breakthrough curves involved the use of a syringe pump (see Fig.14) to drive a sample of $500 \mathrm{ngml}^{-1}$ corticosteroids through a $10 \mathrm{mg}$ electrospun polystyrene fiber sorbent bed at $0.1 \mathrm{mlmin}^{-1}$ with subsequent HPLC-DAD detection. 


\begin{tabular}{lccccccc}
\hline Analyte & \multicolumn{1}{l}{$\begin{array}{l}V_{B}(\mu \mathrm{l}) \\
\text { Observed }\end{array}$} & $\begin{array}{l}V_{B}(\mu \mathrm{l}) \\
\text { Calculated }\end{array}$ & $V_{M}(\mu \mathrm{l})$ & $V_{R}(\mu \mathrm{l})$ & $N_{\text {cal }}$ & $N_{\text {obs }}$ & $k$ \\
\hline Betamethasone & 1400 & 848.18 & 5185.39 & 3044.89 & 7.69 & 13.71 & -0.41 \\
\hline Dexamethasone & 1400 & 924.53 & 5189.44 & 3100.98 & 8.12 & 13.29 & -0.40 \\
\hline
\end{tabular}

Table 2. Chromatographic parameters of the electrospun polystyrene fiber based micro colum sorbent bed format derived from the sigmoid Boltzmann model

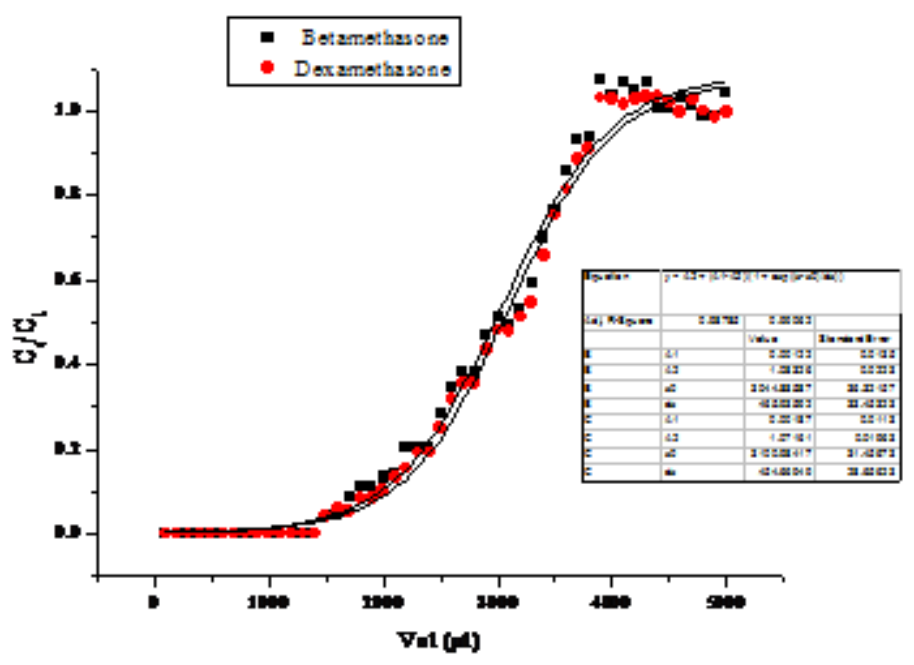

Figure 18. Breakthrough curves determined for two steroids on electrospun polystyrene fibers packed in micro column sorbent bed format presented as lines of best fit using the sigmoid Boltzmann model.

The breakthrough volumes, equilibrium volumes, retention volumes, theoretical plates and retention factor values for betamethasone and dexamethasone were very close which was consistent with the closeness of their $\log \mathrm{P}$ values. This consistency was observed for the breakthrough curves fitted using both the Boltzmann and the Weibull five parameter model. On that basis, it may be concluded that these mathematical models would be suitable for characterisation of any electrospun fiber sorbent-analyte system that follows a similar profile.

Comparison of the calculated theoretical plates for the disk (1.39-2.82) and the micro column (7.98-9.1) SPE devices revealed that the shape of the breakthrough curve could be related to the theoretical plates $[80,91]$. In addition, the observed breakthrough volumes for the disk $(400-500 \mu \mathrm{l})$ and micro column $(1400 \mu \mathrm{l})$ respectively were consistent with the theoretical plates. This may mean that theoretical plates could be important for the retention characteristics of a SPE sorbent bed.

Given the fact that theoretical plates are a function of the available surface area for analyte interaction, it could be concluded that a sorbent material with a larger surface area may exhibit 
a larger number of theoretical plates and consequently a large retention capacity as mass transfer kinetics would be enhanced. Therefore, a larger number of theoretical plates would correspond to a steeper slope of the breakthrough curve as a result of fast mass transfer kinetics.

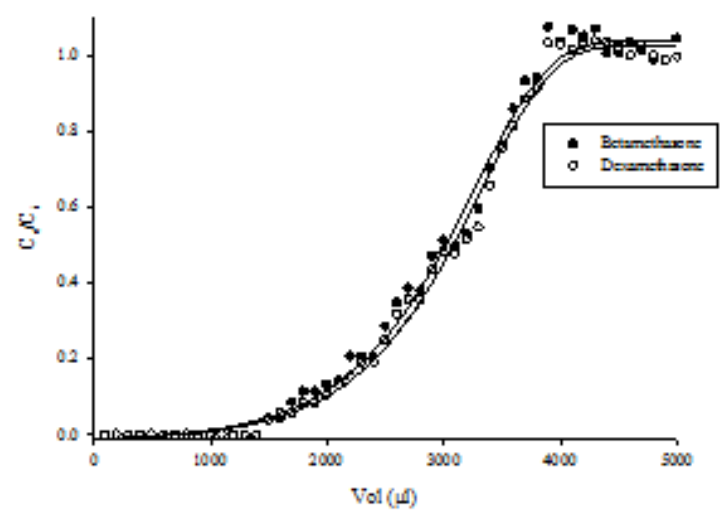

Figure 19. Breakthrough curves determined for two steroids on electrospun polystyrene fibers packed in microcolumn sorbent bed format presented as lines of best fit using the Weibull five parameter model.

\begin{tabular}{|c|c|c|c|c|c|c|}
\hline \multirow[b]{2}{*}{ Analyte } & \multicolumn{5}{|c|}{ Coefficient } & \multirow{2}{*}{$\mathbf{R}^{2}$} \\
\hline & $a$ & b & c & $x_{0}$ & $y_{\circ}$ & \\
\hline \multirow[t]{2}{*}{ Betamethasone } & $1.0396(0.01)$ & 545252504.47 & 879140.39 (7.94) & $3088.50(40.73)$ & $-0.0143(0.0094)$ & 0.9953 \\
\hline & & $(0.30)$ & & & & \\
\hline \multirow[t]{2}{*}{ Dexamethasone } & 1.0528 & 15042770.75 & $23676.51(7.37)$ & 3032.19 (34.33) & $-0.0159(0.0110)$ & 0.9943 \\
\hline & $(0.02)$ & (8.91) & & & & \\
\hline
\end{tabular}

Table 3. Regression parameters for the Weibull five parameter model

\begin{tabular}{llllllll}
\hline Analyte & $\begin{array}{l}V_{B}(\boldsymbol{\mu l}) \\
\text { Observed }\end{array}$ & $\begin{array}{l}V_{B}(\boldsymbol{\mu l}) \\
\text { Calculated }\end{array}$ & $V_{M}(\boldsymbol{\mu l})$ & $V_{R}(\boldsymbol{\mu l})$ & $N_{c a l}$ & $N_{\text {obs }}$ & $k$ \\
\hline Betamethasone & 1400 & 901 & 4250 & 3088.5 & 7.98 & 13.38 & -0.27 \\
\hline Dexamethasone & 1400 & 1020 & 4240 & 3032.2 & 9.1 & 13.80 & -0.28 \\
\hline
\end{tabular}

Table 4. Chromatographic parameters of the electrospun polystyrene fiber based micro column sorbent bed format derived from the Weibull five parameter model.

\section{Conclusions}

Given the fact that the feasibility of electrospun nanofiber based SPE has been successfully demonstrated, it is proposed that future research efforts should be channelled towards 
simplifying SPE device fabrication and SPE procedures. In addition, more research efforts should be channelled towards physicochemical characterisation of electrospun nanofibers and relating to retention characteristics of the electrospun nanofiber based sorbent beds. Therefore, it is expected that with a better control of nanofiber orientation, packing procedures and pore structure, analytically useful electrospun nanofiber based chromatographic sorbent beds for HPLC could be fabricated.

By describing the wide nanofiber sorbent range of chemistries, functionalities and morphologies, it is hoped that this book chapter has provided enough evidence to support the hypothesis that electrospun nanofibers will be an effective class of SPE sorbents.

However several questions relating to the role of electrospinning, surface area to volume ratio, sorptive capacity and the miniaturisation route (should it be SPE or SPME variations?) still need to be answered. Looking into the future one wonders if nanostructured materials will mark the end of the sorbent technology development chain. In conclusion one can say with some degree of confidence that through electrospinning, a new class of sorbent based techniques/devices will eventually find their way into the analytical process either as a low or high resolution separation step.

\section{Acknowledgements}

Sasol is acknowledged for Samuel Chigome's Post Doctoral Research fellowship. Water Research Commission of South Africa, National Research Foundation of South Africa, Agilent Technologies, African Network of Analytical Chemists (SEANAC) and Rhodes University are acknowledged for financial and equipment support.

\section{Author details}

Samuel Chigome* and Nelson Torto*

*Address all correspondence to: n.torto@ru.ac.za

Department of Chemistry, Rhodes University, South Africa

\section{References}

[1] I. Liska, J. Chromatogr., A 885 (2000) 3-16.

[2] J. K. Lokhnauth, Solid phase microextraction and stir bar sorptive extraction coupled to ion mobility spectrometry. 2005. p. 201 pp.

[3] H. Braus, F. M. Middleton, G. Walton, Anal. Chem. 23 (1951) 1160-4. 
[4] S. Chigome, G. Darko, N. Torto, Analyst 136 (2011) 2879-2889

[5] E. D.H., Manual of symbols and terminology for physicochemical quantities and units. Appendix II. Definitions, terminology, and symbols in colloid and surface chemistry, in Pure Appl. Chem. 1972. p. 577-638.

[6] R. P. Schwarzenbach, P. M. Gschwend, D. M. Imboden, Environmental Organic Chemistry. 1992: Wiley. 681 pp.

[7] B. S. Bahl, G. D. Tuli, A. Bahl, Essentials of Physical Chemistry, 24th Edition. 2000: S. Chand \& Co. Ltd. 880 pp.

[8] J. Fraissard, C. W. Conner, Editors, Physical Adsorption: Experiment, Theory and Applications. (Proceedings of the NATO Advanced Study Institute, held 19 May-1 June 1996, in La Colle sur Loup, France.) [In: NATO ASI Ser., Ser. C, 1997; 491]. 1997: Kluwer. 619 pp.

[9] M. C. Hennion, J. Chromatogr., A 856 (1999) 3-54.

[10] K.-U. Goss R. P. Schwarzenbach, Environ. Sci. Technol. 35 (2001) 1-9.

[11] P. W. Lankford, W. W. Eckenfelder, Jr., Editors, Toxicity Reduction in Industrial Effluents. 1990: Van Nostrand Reinhold. 350 pp.

[12] W. J. Thomas B. Crittenden, Adsorption Technology and Design. 1997: Butterworth-Heinemann. $176 \mathrm{pp}$.

[13] S. Mitra J. D. Winefordner, Sample Preparation Techniques in Analytical Chemistry. 2003: John Wiley \& Sons Inc. 400 pp.

[14] R. P. Belardi J. B. Pawliszyn, Water Pollut. Res. J. Can. 24 (1989) 179-191.

[15] S. Risticevic, V. H. Niri, D. Vuckovic, J. Pawliszyn, Anal. Bioanal. Chem. 393 (2009) 781.

[16] F. M. Musteata J. Pawliszyn, TrAC Trends in Analytical Chemistry 26 (2007) 36-45.

[17] D. Vuckovic, X. Zhang, E. Cudjoe, J. Pawliszyn, Journal of Chromatography A 1217 (2010) 4041-4060.

[18] L. Muller. Field analysis by SPME. 1999: Royal Society of Chemistry. p.269-283

[19] J. S. Fritz M. Macka, J. Chromatogr., A 902 (2000) 137-166.

[20] J. S. Fritz, Analytical Solid-Phase Extraction. 1999: Wiley. 210 pp.

[21] C. F. Poole, A. D. Gunatilleka, R. Sethuraman, J. Chromatogr., A 885 (2000) 17-39.

[22] R. Lucena, B. M. Simonet, S. Cárdenas, M. Valcárcel, Journal of Chromatography A 1218 (2011) 620-637.

[23] C. K. Liu, K. Lai, W. Liu, M. Yao, R. J. Sun, Polymer International 58 (2009) 1341-1349. 
[24] G. Y. Oh, Y. W. Ju, M. Y. Kim, H. R. Jung, H. J. Kim, W. J. Lee, Science of the Total Environment 393 (2008) 341-347.

[25] W. G. Shim, C. Kim, J. W. Lee, J. J. Yun, Y. I. Jeong, H. Moon, K. S. Yang, Journal of Applied Polymer Science 102 (2006) 2454-2462.

[26] N. N. Bui, B. H. Kim, K. S. Yang, M. E. Dela Cruz, J. P. Ferraris, Carbon 47 (2009) 2538-2539.

[27] R. Lucena, B. M. Simonet, S. CÃ $j$ rdenas, M. ValcÃ $j$ rcel, Journal of Chromatography A 1218 620-637.

[28] N. Masque, R. M. Marce, F. Borrull, TrAC, Trends Anal. Chem. 17 (1998) 384.

[29] C. Shao, H. Kim, J. Gong, D. Lee, Nanotechnology 13 (2002) 635.

[30] S. S. Choi, S. G. Lee, S. S. Im, S. H. Kim, Y. L. Joo, Journal of Materials Science Letters 22 (2003) 891-893.

[31] A. C. Patel, S. Li, C. Wang, W. Zhang, Y. Wei, Chem. Mater. 19 (2007) 1231-1238.

[32] D. Qi, X. Kang, L. Chen, Y. Zhang, H. Wei, Z. Gu, Anal Bioanal Chem 390 (2008) 929-38.

[33] Z. Liu, X. Kang, F. Fang, Microchim. Acta 168 (2010) 59-64.

[34] X. Kang, C. Pan, Q. Xu, Y. Yao, Y. Wang, D. Qi, Z. Gu, Anal Chim Acta 587 (2007) 75-81.

[35] Y. Zhang, X. Kang, L. Chen, C. Pan, Y. Yao, Z. Z. Gu, Analytical and Bioanalytical Chemistry 391 (2008) 2189-2197.

[36] X. J. Kang, L. Q. Chen, Y. Y. Zhang, Y. W. Liu, Z. Z. Gu, Journal of Separation Science 31 (2008) 3272-3278.

[37] S. Chigome, G. Darko, U. Buttner, N. Torto, Anal. Methods 2 (2010) 623-626.

[38] L.-Q. Chen, X.-J. Kang, J. Sun, J.-J. Deng, Z.-Z. Gu, Z.-H. Lu, J. Sep. Sci. 33 (2010) 2369-2375.

[39] Y. Wang, L. Chen, X. Kang, Q. Xu, Z. Gu, Zhongguo Yaolixue Tongbao 23 (2007) 832.

[40] X. Kang, H. Wei, Y. Zhang, L. Chen, Z. Gu. Progress on Post-Genome Technologies, Proceedings of the International Forum on Post-Genome Technologies (4' IFPT), 4th, Hangzhou, China, Sept. 25-26, (2006) 241-245

[41] F. Fang, X. J. Kang, Z. Y. Liu, Y. Q. Ma, Z. Z. Gu, Chinese Chemical Letters 20 (2009) 1491-1494.

[42] Q. Xu, S. Y. Wu, M. Wang, X. Y. Yin, Z. Y. Wen, W. N. Ge, Z. Z. Gu, Chromatographia 71(2010) 487-492.

[43] Q. Xu, X. Yin, S. Wu, M. Wang, Z. Wen, Z. Gu, Microchimica Acta 168 (2010) 267-275. 
[44] Q. Xu, N. Zhang, X. Yin, M. Wang, Y. Shen, S. Xu, L. Zhang, Z. Gu, J Chromatogr B Analyt Technol Biomed Life Sci 878 (2010) 2403-8.

[45] K. Yoshimatsu, L. Ye, J. Lindberg, I. S. Chronakis, Biosens Bioelectron 23 (2008) 1208-15.

[46] C.-h. Zheng, F.-g. Meng, X.-r. Mo, D.-y. Zhao, M.-m. Yang, Fenxi Shiyanshi 29 (2010) 80-84.

[47] C. Zheng, L. Yang, Z. Yao, D. Zhao, M. Yang, Fenxi Ceshi Xuebao 28 (2009) 926-930.

[48] Z. Yao, L. Yang, C. Zheng, M. Yang, Huaxue Tongbao 72 (2009) 845-848.

[49] L. Zhang Y.-L. Hsieh, Nanotechnology 17 (2006) 4416-4423.

[50] C. L. Casper, J. S. Stephens, N. G. Tassi, D. B. Chase, J. F. Rabolt, Macromolecules 37 (2004) 573-578.

[51] A. Gupta, C. D. Saquing, M. Afshari, A. E. Tonelli, S. A. Khan, R. Kotek, Macromolecules 42 (2009) 709-715.

[52] Q. Shi, N. Vitchuli, L. Ji, J. Nowak, M. McCord, M. Bourham, X. Zhang, J. Appl. Polym. Sci. 120 (2011) 425.

[53] N. Fontanals, R. M. Marce, F. Borrull, TrAC, Trends Anal. Chem. 24 (2005) 394-406.

[54] N. Fontanals, R. M. Marce, F. Borrull, Journal of Chromatography A 1152 (2007) 14-31.

[55] A. Kloskowski, M. Pilarczyk, A. Przyjazny, J. NamieÅ^nik, Critical Reviews in Analytical Chemistry 39 (2009) 43-58.

[56] L. S. Wan, J. Wu, Z. K. Xu, Macromolecular Rapid Communications 27 (2006) 1533-1538.

[57] E. Turiel A. Martin-Esteban, Anal. Chim. Acta 668 (2010) 87.

[58] I. S. Chronakis, B. Milosevic, A. Frenot, L. Ye, Macromolecules 39 (2006) 357-361.

[59] S. Chigome N. Torto, TrAC, Trends Anal. Chem. 38 (2012) 21-31.

[60] X. Kang, C. Pan, Q. Xu, Y. Yao, Y. Wang, D. Qi, Z. Gu, Analytica Chimica Acta 587 (2007) 75-81.

[61] F. Fang, X. J. Kang, Z. Y. Liu, Y. Q. Ma, Z. Z. Gu, Chinese Chemical Letters 20 (2009) 1491-1494.

[62] Z. Liu, X. Kang, F. Fang, Microchimica Acta 168 (2009) 59-64.

[63] X.-J. Kang, L.-Q. Chen, Y. Wang, Y.-Y. Zhang, Z.-Z. Gu, Biomedical Microdevices 11 (2009) 723-729. 
[64] Y. Zhang, X. Kang, L. Chen, C. Pan, Y. Yao, Z.-Z. Gu, Analytical and Bioanalytical Chemistry 391 (2008) 2189-2197.

[65] D. Qi, X. Kang, L. Chen, Y. Zhang, H. Wei, Z. Gu, Anal. Bioanal. Chem. 390 (2008) 929-938.

[66] X.-j. Kang, L.-q. Chen, Y.-y. Zhang, Y.-w. Liu, Z.-z. Gu, Journal of Separation Science 31 (2008) 3272-3278.

[67] J. S. Fritz J. J. Masso, J. Chromatogr., A 909 (2001) 79-85.

[68] S. Chigome, G. Darko, U. Buttner, N. Torto, Analytical Methods 2 (2010) 623-626.

[69] Q. Xu, S.-Y. Wu, M. Wang, X.-Y. Yin, Z.-Y. Wen, W.-N. Ge, Z.-Z. Gu, Chromatographia 71 (2009) 487-492.

[70] Q. Xu, X. Yin, M. Wang, H. Wang, N. Zhang, Y. Shen, S. Xu, L. Zhang, Z. Gu, Journal of Agricultural and Food Chemistry 58 (2010) 11311-11317.

[71] W. Shu-Yan, X. Qian, C. Tian-Shu, W. Min, Y. Xue-Yan, Z. Ni-Ping, S. Yan-Yan, W. Zuo-Yang, G. Zhong-Ze, Fenxi Huaxue/ Chinese Journal of Analytical Chemistry 38 (2010) 503-507.

[72] Q. Xu, N. Zhang, X. Yin, M. Wang, Y. Shen, S. Xu, L. Zhang, Z. Gu, J. Chromatogr., B: Anal. Technol. Biomed. Life Sci. 878 (2010) 2403-2408.

[73] Q. Xu, X. Yin, Y. Shen, N. Zhang, M. Wang, Z. Gu, Chin. J. Chem. 29 (2011) 567-574.

[74] Q. Xu, X. Yin, S. Wu, M. Wang, Z. Wen, Z. Gu, Microchimica Acta 168 (2010) 267-275.

[75] H. Bagheri, A. Aghakhani, M. Akbari, Z. Ayazi, Analytical and Bioanalytical Chemistry 400 (2011) 3607-3613

[76] H. Bagheri, Z. Ayazi, A. Aghakhani, N. Alipour, J. Sep. Sci. 35 (2012) 114-120.

[77] M. Abdel-Rehim, J. Chromatogr., A 1217 (2010) 2569-2580.

[78] G. Darko, S. Chigome, Z. Tshentu, N. Torto, Anal. Lett. 44 (2011) 1855-1867.

[79] C. E. Werkhoven-Goewie, U. A. T. Brinkman, R. W. Frei, Anal. Chem. 53 (1981) 2072-80.

[80] P. Lövkvist J. Å. Jönsson, Analytical Chemistry 59 (1987) 818-821.

[81] K. Bielicka-Daszkiewicz A. Voelkel, Talanta 80 (2009) 614-621.

[82] R. Ferrer, J. L. Beltran, J. Guiteras, Anal. Chim. Acta 346 (1997) 253-258.

[83] M. Mihaly, E. S. Andreiadis, E. Pincovschi, Sci. Bull. - Univ. "Politeh." Bucharest, Ser. B 67 (2005) 57-64.

[84] E. Bacalum, M. Radulescu, E.-E. Iorgulescu, V. David, Rev. Roum. Chim. 56 (2011) 137-143. 
[85] S. V. Romanenko A. G. Stromberg, J. Anal. Chem. 55 (2000) 1024-1028.

[86] S. D. Kolev, Anal. Chim. Acta 229 (1990) 183-9.

[87] W. D. Hillis, Curr Biol 3 (1993) 79-81.

[88] V. F. Pozhidaev, Y. B. Rubinshtein, G. Y. Golberg, S. A. Osadchii, Chem. Pet. Eng. 45 (2009) 460-467.

[89] M. Ramirez C, M. Pereira da Silva, S. G. Ferreira L, O. Vasco E, Journal of Hazardous Materials 146 (2007) 86-90.

[90] J. E. Clark S. V. Olesik, Anal. Chem. (Washington, DC, U. S.) 81 (2009) 4121.

[91] G. I. Senum, Environ. Sci. Technol. 15 (1981) 1073-5. 
